\title{
ASSOCIATION OF INFRARED DARK CLOUD CORES WITH YSOS: STARLESS OR STARRED IRDC CORES
}

\author{
Gwanjeong Kim ${ }^{1,2,5}$, Chang Won Lee ${ }^{1,2,6}$, Jongsoo Kim ${ }^{1,2}$, Youngung Lee ${ }^{1}$, Javier Ballesteros-Paredes ${ }^{3}$, \\ Philip C. Myers ${ }^{4}$, AND S. Kurtz ${ }^{3}$ \\ ${ }^{1}$ International Center for Astrophysics, Korea Astronomy and Space Science Institute, 61-1 Hwaam-dong, \\ Yuseong-gu, Daejeon 305-348, Korea \\ 2 Department of Astronomy and Space Science, University of Science \& Technology, 113 Gwahangno, Yuseong-gu, \\ Daejeon 305-333, Korea \\ 3 Centro De Radioastronomía y Astrofísica, Universidad Nacional Autónoma De México, Morelia, Michoacán \\ 58089, México \\ 4 Harvard-Smithsonian Center for Astrophysics, 60 Garden Street, Ms 42, Cambridge, Ma 02138, USA \\ 5 E-mail: archer81@kasi.re.kr6 cwl@kasi.re.kr \\ (Received December 04, 2009; Accepted December 28, 2009)
}

\begin{abstract}
In this paper we examined the association of InfraRed Dark Cloud (IRDC) cores with YSOs and the geometric properties of the IRDC cores. For this study a total of 13,650 IRDC cores were collected mainly from the catalogs of the IRDC cores published from other studies and partially from our catalog of IRDC cores containing new 789 IRDC core candidates. The YSO candidates were searched for using the GLIMPSE, MSX, and IRAS point sources by the shape of their SED or using activity of water or methanol maser. The association of the IRDC cores with these YSOs was checked by their lineof-sight coincidence within the dimension of the IRDC core. This work found that a total of 4,110 IRDC cores have YSO candidates while 9,540 IRDC cores have no indication of the existence of YSOs. Considering the 12,200 IRDC cores within the GLIMPSE survey region for which the YSO candidates were determined with better sensitivity, we found that 4,098 IRDC cores $(34 \%)$ have at least one YSO candidate and 1,072 cores among them seem to have embedded YSOs, while the rest 8,102 (66\%) have no YSO candidate. Therefore, the ratio of [N(IRDC core with protostars)]/[N(IRDC core without YSO)] for 12,200 IRDC cores is about 0.13. Taking into account this ratio and typical lifetime of high-mass embedded YSOs, we suggest that the IRDC cores would spend about $10^{4} \sim 10^{5}$ years to form high-mass stars. However, we should note that the GLIMPSE point sources have a minimum detectable luminosity of about $1.2 \mathrm{~L} \odot$ at a typical IRDC core's distance of $\sim 4 \mathrm{kpc}$. Therefore, the ratio given here should be a lower limit and the estimated lifetime of starless IRDC cores can be an upper limit. The physical parameters of the IRDC cores somewhat vary depending on how many YSO candidates the IRDC cores contain. The IRDC cores with more YSOs tend to be larger, more elongated, and have better darkness contrast than the IRDC cores with fewer or no YSOs.
\end{abstract}

Key words : catalogs — dust, extinction - galaxy: general — infrared: ISM - ISM: clouds — ISM: structure

\section{INTRODUCTION}

How do massive stars form? Do they form in a similar way to how low-mass stars do? This is a question that many astronomers would like to frequently ask, but can hardly answer for. One of suggested scenarios in the early stage of high-mass star formation (e.g., Beuther et al. 2007) is that some of low/intermediatemass protostars form in High-Mass Starless Cores (HMSCs) through an accretion process suggested by the standard theory of low-mass star formation (e.g., Shu et al. 1987) and become massive $\left(>8 \mathrm{M}_{\odot}\right)$ either through massive accretion $\left(10^{-4}-10^{-3} \mathrm{M}_{\odot} \mathrm{yr}^{-1}\right)$ (e.g., Nor-

Corresponding Author: G. KIM berg \& Maeder 2000) or through coalescence process (e.g., Bally \& Zinnecker 2005). Then High-Mass Cores harboring Low/Intermediate protostars turn to be Protostellar Objects (HMPOs). When the processes obtaining matter stop, the HMPOs are expected to finally become high-mass stars. In this scenario, however, it is mostly unknown how the HMSCs form and how the accreting low/intermediate-mass stars really grow to high-mass stars, partly because there are relatively less studies on the HMSCs and the High-Mass Cores with protostars compared with low mass cores.

Last decade, however, has seen a significant improvement on the studies of high-mass star forming regions due to the surveys made with the Infrared Space Observatory (ISO; Perault et al. 1996; Hennebelle et al. 
2001), the Mid-course Space eXperiment (MSX; Price 1995; Egan et al. 1998), and the Spitzer Space Telescope (SST; Benjamin et al. 2003) which discovered a number of the InfraRed Dark Clouds (IRDCs) seen in absorption against the bright, diffuse mid-infrared Galactic background.

The SST, especially, provided new eyes to enable to penetrate the IRDCs of a few hundreds $A_{v}$ to see newly born stars there. The IRDCs have large dust column densities resulting in high extinction and thus they do not exhibit detectable emission at 8-25 $\mu \mathrm{m}$ (Carey et al. 1998). While nearby dark dense molecular cores are often the site of the low-mass star formation, the IRDCs are believed to be the natal sites of mostly massive stars. Their overall features are known from recent studies of dust continuum and molecular line emission at (sub)mm wavelengths: They are usually distant $(1-8 \mathrm{kpc})$, large $(\sim 1-3 \mathrm{pc})$, dense $\left(10^{2} \sim 10^{4}\right.$ $\left.\mathrm{cm}^{-3}\right)$, and massive $\left(10^{2}-10^{4} \mathrm{M}_{\odot}\right)$ (e.g., Rathborne et al. 2006; Simon et al. 2006b).

In most case the IRDCs have substructures showing locally mid-IR extinction peak due to their higher column density compared with other region of the IRDC, which are called as IRDC cores. These IRDC cores have typical sizes of $0.02 \sim 0.8 \mathrm{pc}$, masses of $10 \sim 10^{3}$ $\mathrm{M}_{\odot}$, and densities of $10^{3} \sim 10^{7} \mathrm{~cm}^{-3}$ (Rathborne et al. 2006). Some IRDC cores without an embedded source have lower temperature $\left(\mathrm{T}_{\mathrm{NH}_{3}} \sim 16 \mathrm{~K}\right)$, narrow line-width $\left(\Delta \mathrm{V}_{\mathrm{NH}_{3}} \sim 1.6 \mathrm{~km} / \mathrm{s}\right)$, lack of strong complex molecule emission, and lower detection rate $(\sim 12 \%)$ of water masers, representing a less active environment than HMPOs and Ultracompact H II regions (Sridharan et al. 2002, 2005; Wang et al. 2006: Churchwell et al. 1990). On the other hand other IRDC cores with embedded sources have warmer temperature $(\sim 25 \mathrm{~K})$, shocked gas (e.g. $\mathrm{H}_{2}$ or $\left.\mathrm{SiO}\right)$, broad linewidth $\left(\Delta \mathrm{V}_{\mathrm{HCN}(4-3)} \sim 10 \mathrm{~km} / \mathrm{s}\right)$, rich complex molecular spectrum (e.g. $\mathrm{CH}_{3} \mathrm{CH}_{2} \mathrm{CN}$ ), and more noticeable methanol/water maser activities (Redman et al. 2003; Ormel et al. 2005; Rathborne et al. 2005, 2007, 2008; Pillai et al. 2006; Ragan et al. 2006; Wang et al. 2006).

These properties remind us that the IRDC cores without any embedded source are analogous to lowmass starless cores, while the IRDC cores with embedded sources mimic the properties of star-containing low-mass cores. The characteristics of IRDCs are similar to those of HMSCs or High-Mass Cores with protostars on the core scale (sub-parsec scale), or those of Massive Starless Clumps or Protocluster on the clump/cluster scale (a few parsec scale) (Rathborne et al. 2006; Beuther et al. 2007). The HMSCs are believed to preserve the initial conditions (e.g., core mass function) for high-mass star formation until they turn to be the High-Mass Cores containing protostars which mostly lose their initial information by the strong radiation and stellar wind of embedded massive protostars. Hence, it is clear that the IRDC cores are of prime importance to study the early processes of high-mass star formation.

Constructing a fully possible set of IRDC cores and examining an association of the IRDC cores with YSOs should be an important first step for a systematic study of their role in high-mass star formation. For this purpose we collect all possible IRDC cores from previously published catalogs including our subset of IRDCs that we found in this study and examine association of those with any possible embedded YSOs *.

Recent Galactic Legacy Infrared Mid-Plane Survey (GLIMPSE) made with SST was sensitive enough to detect about 70 million point sources along the line of sight of Galactic regions having the IRDC cores. Combining catalogs of the IRDC cores and GLIMPSE point sources, it seems now possible to make a systemic study of the association of the IRDC cores with YSOs which will be a basis of further study on the early process in high-mass star formation.

In this paper we collect most of the IRDC cores studied previously by other researchers with a subset of our new IRDC cores which are missed in other cata$\operatorname{logs}$ to examine in all these IRDC cores the existence of YSO candidates such as the GLIMPSE, MSX, and IRAS point sources, and water and methanol masers.

In $\S$ II we describe how we collect the IRDC cores known and how we identify new IRDC cores. In $\S$ III we examine the association of the IRDC cores with YSOs and then in $\S \mathrm{IV}$ we discuss various statistics of geometric properties of the IRDC cores with and without YSOs. We summarize our results in $\S \mathrm{V}$.

\section{COLLECTION OF THE INFRARED DARK CLOUD CORES}

\section{(a) The IRDC Cores from Literatures}

One of the most comprehensive collection of the IRDC cores is a catalog by Simon et al. (2006a, hereafter SJRC), consisting of 12,744 IRDC cores. Before the SJRC catalog was published, there have been also many other studies on the IRDCs and their cores using (sub)mm molecular line and continuum observations (e.g., Carey et al. 1998, 2000; Teyssier et al. 2002; Fiege et al. 2004; Garay et al. 2004; Ormel et al. 2005; Beuther et al. 2005; Pillai et al. 2006). Sridharan et al. (2005) observed over 50 IRDC cores in 1.2 $\mathrm{mm}$ continuum and $\mathrm{NH}_{3}$ molecular line, finding that most of them were high-mass starless cores. Recently, Rathborne et al. (2006) and Ragan et al. (2006) significantly increased the number of IRDC cores studied to over 150. From all the studies in (sub)mm molecular lines or continuum, we collect a sample of 87 IRDC cores which are not listed in the SJRC catalog.

\footnotetext{
A vast catalog of Spitzer Dark Clouds was recently released (Peretto \& Fuller 2009) after this work was being completed. Although it lists more dark clouds than we collected, our collection of IRDCs have twice wider coverage of the galaxy than SST GLIMPSE survey area and will be a reasonable sample to discuss any statistics related with IRDCs.
} 
TABLE 1

Statistics OF THE ASSOCIATION OF IRDC CORES With YSO CANDIDATES

\begin{tabular}{rrr}
\hline \hline $\begin{array}{c}\text { \# of YSOs } \\
\text { /YSO candidates }\end{array}$ & $\begin{array}{c}\text { \# of IRDC cores } \\
\text { (All Sample) }\end{array}$ & $\begin{array}{c}\text { \# of IRDC cores } \\
\text { (GLIMPSE sample) }\end{array}$ \\
\hline more than 2 YSOs & 2,108 & 2,104 \\
1 YSO & 2,002 & 1,994 \\
None & 9,540 & 8,102 \\
\hline GLIMPSE Point Source & 4,036 & 4,028 \\
IRAS Point Source & 172 & 168 \\
MSX Point Source & 69 & 68 \\
$\mathrm{H}_{2}$ O maser & 8 & 33 \\
Methanol maser & 33 & 1,072 \\
EYSO & 1,077 & 3,593 \\
PMS & 3,601 & \\
\hline
\end{tabular}
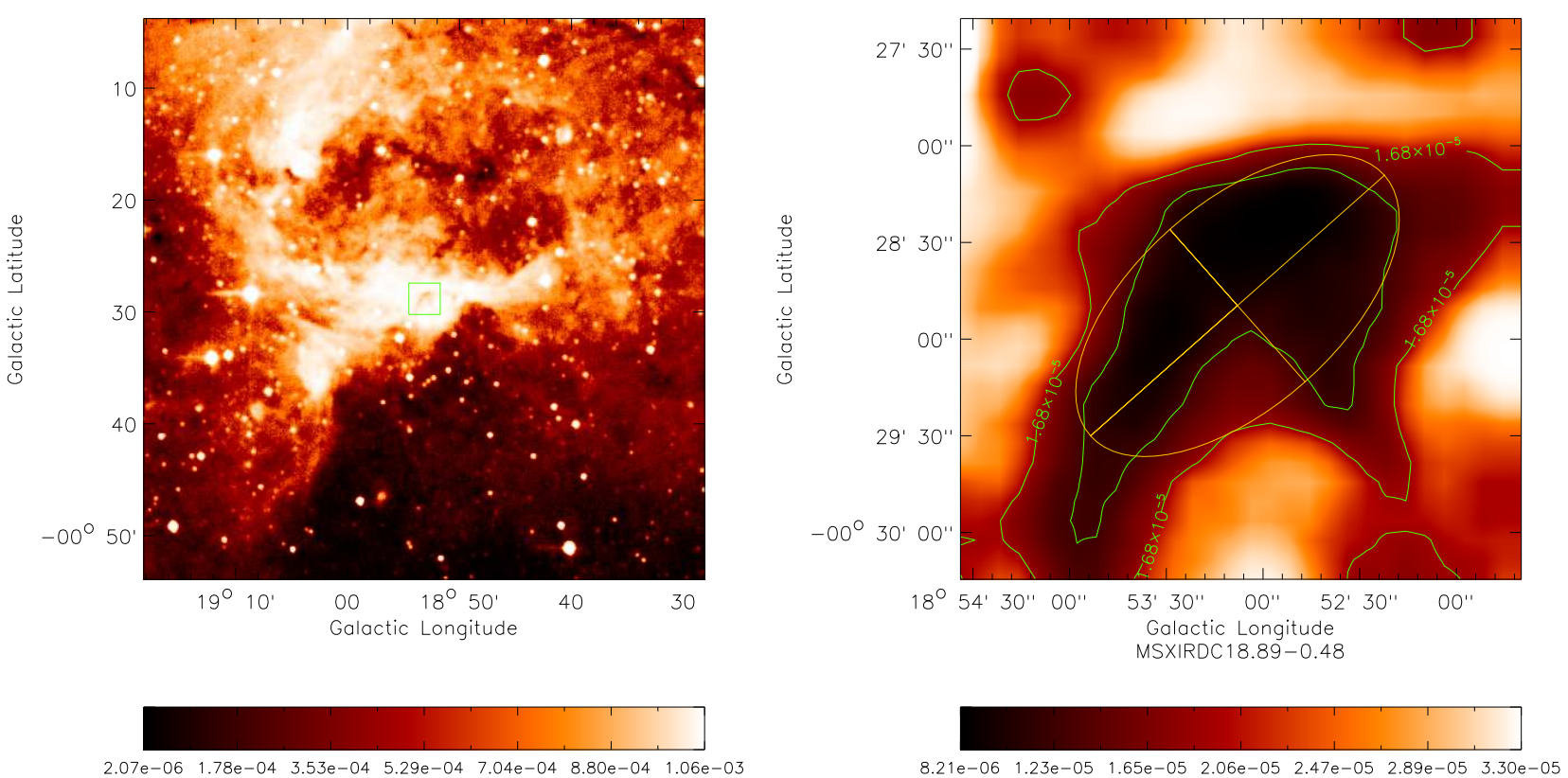

Fig. 1. - An example of how to select our new IRDC core candidates. The image of an IRDC core candidate (MSXIRDC18.89-0.48) on the left is an $8.28 \mu \mathrm{m}$ image of $50^{\prime} \times 50^{\prime}$, the contrast of which is enhanced with the function of histogram equalization. The other image on the right is a blow-up of the green box in central region of the left panel. The FWHMin brightness and the background brightness levels are $1.2 \times 10^{-5}$ and $1.6 \times 10^{-5} \mathrm{Wm}^{-2} \mathrm{sr}^{-1}$, respectively. An ellipse overlays on the FWHMin contour.

\section{(b) Newly Selected IRDC Core Candidates}

The IRDC cores in the SJRC catalog have been selected using an automatic algorithm finding extinction regions (the IRDC cores) of high contrast with respect to the model background in the MSX 8.28 micron images. However, even though the SJRC catalog was able to include most of the IRDC cores seen in the Galactic plane, their algorithm resulted in missing compact dark sources having poor contrast or small regions sur- 
rounded by the very bright mid-infrared emission of the background (Simon et al. 2006). Here we present our additional efforts to add more IRDC cores which were missed in the SJRC catalog, by making the contrast enhancement of images with the function of histogram equalization $^{\dagger}$ on the IDL program ${ }^{\ddagger}$. Our IDL scripts using this technique enable to load MSX $8.28 \mu \mathrm{m}$ images and enhance the darkness contrast of the IRDC core candidates embedded in the bright Galactic background to identify them by eye inspection. We searched for all MSX A band images for the entire Galactic plane $\left(|1|<180^{\circ},|\mathrm{b}|<5^{\circ}\right)$ which are retrievable in fits format up to size of $6^{\circ} \times 6^{\circ}$ from the NASA/IPAC InfraRed Science Achieve (IRSA) $)^{\S}$. A typical searching size of images was 50 arc min $\times 50$ arc min (Figure 1). First, a dark region in the bright background is searched for by eyes. Then its area is chosen by clicking two diagonal corners with a mouse button and the contrast of the regions is enhanced with the function of histogram equalization in IDL scripts. Once a dark region is selected as an IRDC core candidate sample (Figure 1), some geometrical properties of the IRDC cores are measured. The shapes of the identified IRDC cores are

$\dagger$ Histogram equalization is the technique by which the dynamic range of the histogram of an image is adjusted. Histogram equalization assigns the intensity values of pixels in the input image such that the output image contains a uniform distribution of intensities. It improves contrast and the goal of histogram equalization is to obtain a uniform histogram. See: http://www.codersource.net/csharp_histogram_equalization.aspx $\ddagger$ IDL is a kind of commercial products of ITT Visual Information Solutions and is a software for data analysis, data visualization, and software application development. See http://www.ittvis.com/

$\S$ The IRSA is web-based and machine-friendly tools for efficient access to calibrated science data sets obtained from infrared and submillimeter missions of NASA. See http://irsa.ipac.caltech.edu/

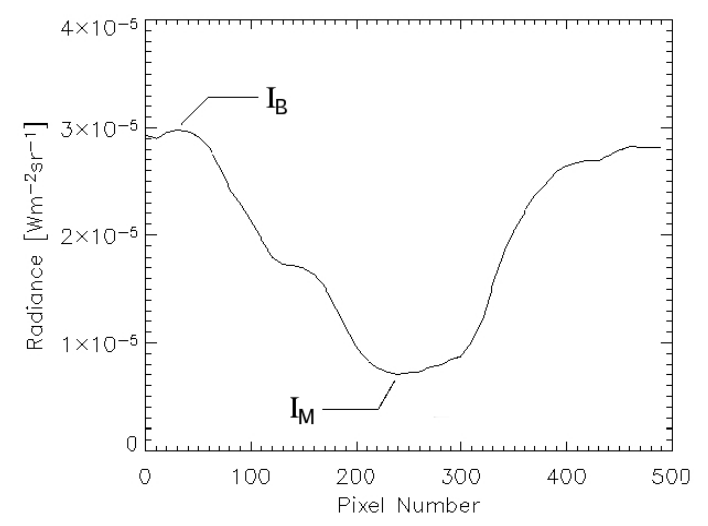

Fig. 2. - A profile-cut obtained by crossing the minimum brightness intensity $\left(\mathrm{I}_{\mathrm{M}}\right)$ of MSXIRDC18.89-0.48. This is useful to estimate the background brightness intensity $\left(\mathrm{I}_{\mathrm{B}}\right)$ of the IRDC which in turn enables us to determine the Full Width at Half Minimum (FWHMin) contour of the IRDC. described by the Full Width at Half Minimum (FWHMin) contours of their intensities. The FWHMin contour level of each region is determined from the background brightness level and the minimum brightness level. Profile-cuts passing through the minimum intensity position were useful in this process, to specify the background brightness level (See Figure 2). We determined the background brightness level of each IRDC core by averaging the brightness over a small circular region at roughly constant background brightness near the part of boundary of IRDC cores. Typically the diameter of these regions was $5^{\prime \prime}$. The selection of the background regions in this way is somewhat arbitrary. We chose seven positions for three IRDC cores as the background candidates to estimate the intensity levels of the selected regions and examine their possible variation. We found a typical deviation of about $10 \%$ in the background intensity value that we adopt. The determination of the background brightness level of the IRDC core enables to specify the level of the FWHMin contour and also the Darkness Contrast (DC) defined as $\frac{\left(I_{B}-I_{M}\right)}{I_{B}}$ where $I_{M}$ is the minimum intensity and $I_{B}$ is background intensity of the IRDC core (Lee \& Myers 1991). We selected only the candidates whose FWHMin of the intensities can make a closed contour so that an ellipse fitting of the contour can be possible. By fitting the FWHMin contour of each IRDC core with an ellipse, we assigned the coordinates of the IRDC core candidates as the central position of the ellipse and derived the geometrical parameters of the IRDC core, such as the angular sizes of the major and minor axes (a and b), Position Angles (PA), and Equivalent Diameter (ED). The latter is defined as $2 \times \sqrt{\frac{A}{\pi}}$, where $\mathrm{A}$ is the area within the FWHMin contour of the IRDC.

From this work, a total of 789 IRDC core candidates were identified $~$. Most of them $(79 \%)$ have typical angular sizes between $0^{\prime} .4$ and $1^{\prime} .3$ with a mean ( \pm s.e.m.: standard error of the mean) of $0^{\prime} .88 \pm 0^{\prime} .02$ and have typical aspect ratios between 1 and 2.2 with a mean ( \pm s.e.m.) of $1.82 \pm 0.02$. Whether these IRDC core candidates are real clouds can be tested with molecular line observations made toward them. We used ${ }^{13} \mathrm{CO}(1-0)$ BU-FCRAO Galactic Ring Survey (GRS) on the Galactic plane of $18^{\circ}<1<55^{\circ}$ and $|\mathrm{b}|<1^{\circ}$ obtained by Jackson et al. (2006) for this purpose and selected 17 IRDC core candidates in the GRS region whose angular size is larger than $2^{\prime}$ (Note that angular resolution of the GRS survey is $\left.46^{\prime \prime}\right)$. We found that the extinction shapes of 11 among the tested samples well coincide with the shape of ${ }^{13} \mathrm{CO}$ contour map (Figure 3), implying that a good fraction (about 65\%) of the IRDC core candidates selected in this study can be real clouds.

\footnotetext{
I About half of these targets are found to be also listed in the new catalog of Spitzer Dark Clouds by Peretto \& Fuller 2009, meaning that the other half of the IRDC cores that we found are new.
} 

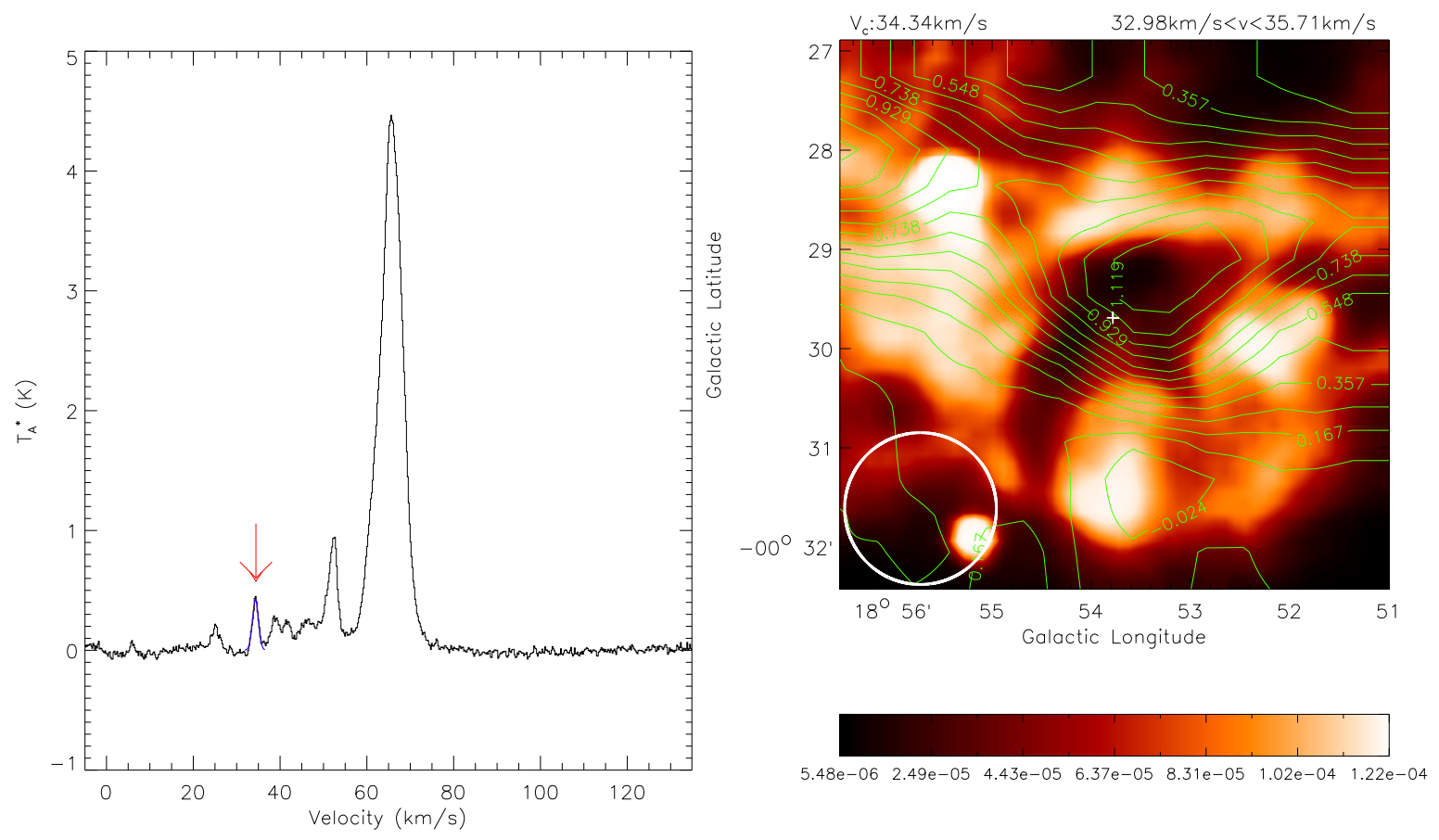

Fig. 3.- Comparison of the extincted image of an IRDC core with ${ }^{13} \mathrm{CO}(1-0)$ molecular line emissions. The ${ }^{13} \mathrm{CO}(1-0)$ profile on the left panel is from the BU-FCRAO Galactic Ring Survey by Jackson et al. (2006). The right panel compares the extincted image of the IRDC with the CO emission, indicating that the distribution of the CO emission at $\sim 34 \mathrm{~km} \mathrm{~s}{ }^{-1}$ coincides with the shape of the IRDC core. The white circle at the left edge of the right panel indicates the beam size $\left(46^{\prime \prime}\right)$ of the telescope for ${ }^{13} \mathrm{CO}(1-0)$ observation .

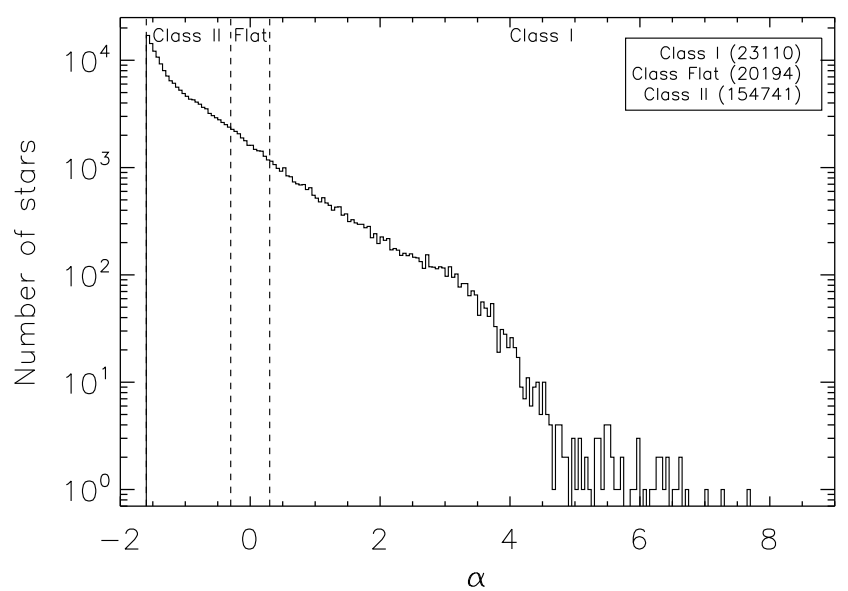

Fig. 4. - A histogram of the slope $(\alpha)$ of the Spectral Energy Distribution from the GLIMPSE point sources. They were classified as Class I $(\alpha \geq 0.3)$, Flat spectrum ($0.3 \leq \alpha<0.3)$, and Class II $(-1.6 \leq \alpha<-0.3)$ following Greene et al. (1994). 198,045 point sources may be EYSOs (Class I objects) or PMSs (Flat spectrum objects or Class II objects).

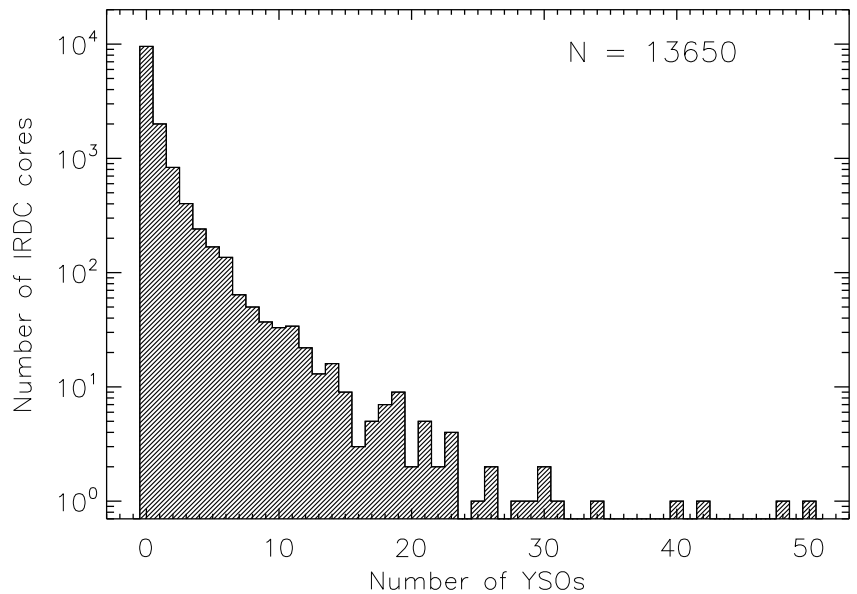

Fig. 5. - A number distribution of 13,650 IRDC cores according to the number of YSO candidates. The IRDC cores without YSO candidate occupy about $70 \%$ of this sample while the IRDC cores with YSO candidates are about $30 \%$. It is interesting to note that about $49 \%$ of the IRDC cores with YSO candidates have a single YSO candidates while others $(\sim 51 \%)$ have multiple YSOs. 
TABLE 2

NEWLY SELECTED 789 IRDC CORES.

\begin{tabular}{|c|c|c|c|c|c|c|c|c|c|c|c|}
\hline $\begin{array}{l}\text { Num. } \\
\text { (1) }\end{array}$ & $\begin{array}{c}\text { Source } \\
(2)\end{array}$ & $\begin{array}{c}\text { R.A. } \\
(2000) \\
(3)\end{array}$ & & $\begin{array}{c}\text { Dec. } \\
(2000 \\
(4)\end{array}$ & & $\begin{array}{c}\mathrm{a} \\
\left({ }^{\prime}\right) \\
(5)\end{array}$ & $\begin{array}{c}\mathrm{b} \\
\left({ }^{\prime}\right) \\
(6)\end{array}$ & $\begin{array}{c}\text { ED } \\
\left({ }^{\prime}\right) \\
(7)\end{array}$ & $\begin{array}{l}\mathrm{PA} \\
\left({ }^{\circ}\right) \\
(8)\end{array}$ & $\begin{array}{l}\mathrm{DC} \\
(9)\end{array}$ & $\begin{array}{l}\mathrm{YSO}^{\mathrm{a}} \\
(10)\end{array}$ \\
\hline 1 & MSXIRDC0.02+0.08 & 174521.7 & -28 & 52 & 45.9 & 0.92 & 0.58 & 07.11 & 071 & 0.18 & \\
\hline 2 & MSXIRDC0.05-0.19 & 174629.6 & -28 & 59 & 20.6 & 1.82 & 0.82 & 10.43 & 354 & 0.19 & $\mathrm{~S}^{I(2), I I(17)}$ \\
\hline 3 & MSXIRDC0.10-0.08 & 174609.8 & -28 & 53 & 23.7 & 1.10 & 0.95 & 10.09 & 332 & 0.35 & $\mathrm{~S}^{I I(3)}, \mathrm{I}^{E(1)}$ \\
\hline 4 & MSXIRDC0.15-0.09 & 174619.6 & -28 & 51 & 15.4 & 1.35 & 0.85 & 10.49 & 034 & 0.26 & $\mathrm{~S}^{I I(5)}$ \\
\hline 5 & MSXIRDC0.17-0.10 & 174625.8 & -28 & 50 & 36.8 & 1.16 & 0.64 & 09.19 & 085 & 0.24 & $\cdots$ \\
\hline 6 & MSXIRDC0.32-0.13 & 174653.4 & -28 & 43 & 47.6 & 0.53 & 0.50 & 06.05 & 091 & 0.39 & $\mathrm{~S}^{I I(1)}$ \\
\hline 7 & MSX IRDC0 $.34+0.10$ & 174601.8 & -28 & 35 & 53.5 & 1.01 & 0.63 & 07.74 & 343 & 0.27 & $\mathrm{~S}^{F(1), I I(3)}$ \\
\hline 8 & MSX IRDC0 $.38-0.69$ & 174913.5 & -28 & 58 & 12.0 & 0.84 & 0.73 & 07.33 & 125 & 0.24 & $\ldots$ \\
\hline 9 & MSXIRDC0.39-0.83 & 174948.1 & -29 & 01 & 59.7 & 0.90 & 0.45 & 06.08 & 326 & 0.22 & .. \\
\hline 10 & MSXIRDC0 $.40-0.15$ & 174708.7 & -28 & 40 & 08.4 & 0.77 & 0.75 & 07.04 & 094 & 0.33 & $\mathrm{~S}^{I I(1)}$ \\
\hline 11 & MSXIRDC0 $.42-0.17$ & 174716.3 & -28 & 39 & 53.8 & 1.03 & 0.57 & 07.51 & 031 & 0.30 & $\mathrm{~S}^{I(1), I I(3)}$ \\
\hline 12 & MSXIRDC0.42-0.26 & 174736.1 & -28 & 42 & 48.1 & 0.64 & 0.59 & 06.06 & 050 & 0.24 & $\mathrm{~S}^{I I(3)}$ \\
\hline 13 & MSXIRDC0 $.46-0.66$ & 174917.2 & -28 & 35 & 03.3 & 1.98 & 1.02 & 13.94 & 332 & 0.43 & $\mathrm{~S}^{F(1)}$ \\
\hline 14 & MSXIRDC0.48-0.69 & 174927.4 & -28 & 53 & 09.0 & 0.58 & 0.36 & 04.47 & 328 & 0.22 & $\mathrm{~S}^{I(2)}$ \\
\hline 15 & MSXIRDC0.53-0.59 & 174911.0 & -28 & 47 & 38.1 & 1.11 & 0.66 & 08.13 & 012 & 0.24 & $\mathrm{~S}^{I I(2)}$ \\
\hline 16 & MSXIRDC0.55-0.66 & 174930.1 & -28 & 48 & 39.8 & 1.21 & 0.48 & 07.37 & 000 & 0.25 & $\mathrm{~S}^{I I(1)}$ \\
\hline 17 & MSXIRDC0 $.58-0.53$ & 174904.0 & -28 & 43 & & 1.63 & 1.12 & 12.10 & 113 & 0.29 & $\mathrm{~S}^{I(1), I I(4)}, \mathrm{M}^{F(1)}$ \\
\hline 18 & MSXIRDC0.59-0.58 & 174917.6 & -28 & 44 & 00.6 & 1.29 & 0.69 & 09.36 & 357 & 0.25 & $\cdots$ \\
\hline \multicolumn{12}{|c|}{ This is a cut-in data } \\
\hline 771 & MSXIRDC359.33-0.09 & 174421.8 & -29 & 33 & 4 & 0.72 & 0.48 & 05.74 & 111 & 0.23 & $\overline{\mathrm{S}^{I I}}$ \\
\hline 772 & MSXIRDC359.36-0.13 & 174435.0 & -29 & 32 & 57.1 & 1.49 & 0.75 & 10.35 & 030 & 0.40 & $\mathrm{~S}^{I(1), I I(8)}$ \\
\hline 773 & MSXIRDC359.39-0.11 & 174433.9 & -29 & 30 & 49.2 & 0.57 & 0.40 & 04.75 & 017 & 0.18 & $\mathrm{~S}^{I I(1)}$ \\
\hline 774 & MSXIRDC359.40-0.59 & 174630.8 & -29 & 45 & 22.6 & 1.75 & 1.34 & 12.72 & 057 & 0.26 & $\mathrm{~S}^{I I(1)}$ \\
\hline 775 & MSXIRDC359.41-0.20 & 174500.7 & -29 & 32 & 37.0 & 0.66 & 0.41 & 05.08 & 338 & 0.17 & \\
\hline 776 & MSXIRDC359.46-0.05 & 174431.4 & -29 & 25 & 33.9 & 1.65 & 0.45 & 07.89 & 347 & 0.21 & $\mathrm{~S}^{F(1), I I(3)}$ \\
\hline 777 & MSXIRDC359.46-0.11 & 174446.1 & -29 & 27 & 36.4 & 0.67 & 0.40 & 05.05 & 016 & 0.31 & $\mathrm{~S}^{I I(2)}$ \\
\hline 778 & MSXIRDC359.53+0.05 & 174418.2 & -29 & 19 & 00.8 & 1.29 & 0.73 & 08.93 & 351 & 0.18 & $\mathrm{~S}^{F(1), I I(3)}$ \\
\hline 779 & MSXIRDC359.55+0.03 & 174424.6 & -29 & 18 & 23.0 & 1.29 & 0.50 & 07.48 & 106 & 0.14 & $\mathrm{~S}^{I I(1)}$ \\
\hline 780 & MSXIRDC359.55+0.00 & 174433.0 & -29 & 18 & 55.6 & 2.37 & 0.54 & 11.16 & 351 & 0.26 & $\mathrm{~S}^{F(1), I I(16)}$ \\
\hline 781 & MSXIRDC359.56-0.21 & 174522.7 & -29 & 24 & 57.8 & 1.50 & 1.24 & 12.24 & 043 & 0.23 & $\mathrm{~S}^{I I(3)}$ \\
\hline 782 & MSXIRDC359.67+0.03 & 174441.4 & -29 & 12 & 14.2 & 2.42 & 0.34 & 08.33 & 020 & 0.10 & $\mathrm{~S}^{I(1), I I(5)}$ \\
\hline 783 & MSXIRDC359.73+0.04 & 174449.4 & -29 & 08 & 51.8 & 2.44 & 0.62 & 11.75 & 017 & 0.19 & $\mathrm{~S}^{F(1), I I(9)}$ \\
\hline 784 & MSXIRDC359.75+0.07 & 174443.7 & -29 & 06 & 44.4 & 0.88 & 0.70 & 06.77 & 076 & 0.25 & $\mathrm{~S}^{I I(2)}$ \\
\hline 785 & MSXIRDC359.80+0.05 & 174456.9 & -29 & 04 & 51.5 & 0.79 & 0.23 & 03.88 & 358 & 0.06 & $\cdots$ \\
\hline 786 & MSXIRDC359.81+0.04 & 174500.6 & -29 & 04 & 27.8 & 0.75 & 0.52 & 05.92 & 039 & 0.14 & $\cdots$ \\
\hline 787 & MSXIRDC359.91+0.11 & 174457.9 & -28 & 57 & 24.3 & 0.81 & 0.29 & 04.47 & 090 & 0.10 & $\cdots$ \\
\hline 788 & MSXIRDC359.94+0.11 & 174503.0 & -28 & 55 & 45.3 & 1.07 & 0.76 & 08.56 & 082 & 0.22 & $\mathrm{~S}^{F(2), I I(12)}$ \\
\hline 789 & MSXIRDC0.00+0.11 & 174511.7 & -28 & 52 & 41.8 & 1.31 & 0.81 & 09.98 & 024 & 0.32 & $\mathrm{~S}^{I I(5)}$ \\
\hline
\end{tabular}

Note.-The full version of this table is available in electronic form. This catalog contains 789 IRDC cores newly selected from this study. In the table sequential numbers of the IRDC cores are given in column 1, the source name in column 2, the coordinates of the IRDC cores in column 3 and 4 as the R.A. and Dec. [J2000], the diameter of the major and minor axes of the IRDC cores in column 5 and 6, the Equivalent Diameter (ED) of the IRDC cores in column 7, the Position Angle (PA) in column 8, the Darkness Contrast (DC) of the IRDC cores in column 9, and possible YSOs association in column 10 .

${ }^{a} \mathrm{H}(\#)$ : water maser, Me(\#): Methanol maser, $\mathrm{I}^{E(\#)}$ : IRAS point source (EYSO), $\mathrm{I}^{P(\#)}$ : IRAS point source (PMS), $\mathrm{M}^{I(\#)}$ : MSX point source (class I), $\mathrm{M}^{F(\#)}$ : MSX point source class (Flat SED source), $\mathrm{M}^{I(\#)}$ : MSX point source (class II), $\mathrm{S}^{I(\#)}$ : SST GLIMPSE point source (class I), $\mathrm{S}^{F(\#)}$ : SST GLIMPSE point source class (Flat SED source), and $\mathrm{S}^{I(\#)}$ : SST GLIMPSE point source (class II), where the number in () indicates the number of YSO candidates. 
TABLE 3

87 IRDC CORES SELECTED FROM LITERATURES.

\begin{tabular}{|c|c|c|c|c|c|c|c|c|c|c|c|c|}
\hline $\begin{array}{l}\text { Num. } \\
\text { (1) } \\
\end{array}$ & $\begin{array}{c}\text { Source } \\
(2) \\
\end{array}$ & $\begin{array}{l}\text { R.A. } \\
(2000) \\
(3) \\
\end{array}$ & & $\begin{array}{r}\text { Dec } \\
(2000 \\
(4) \\
\end{array}$ & & $\begin{array}{c}\mathrm{a} \\
\left({ }^{\prime}\right) \\
(5) \\
\end{array}$ & $\begin{array}{l}\mathrm{b} \\
\left({ }^{\prime}\right) \\
(6) \\
\end{array}$ & $\begin{array}{l}\text { ED } \\
\left({ }^{\prime}\right) \\
(7) \\
\end{array}$ & $\begin{array}{l}\mathrm{PA} \\
\left({ }^{\circ}\right) \\
(8) \\
\end{array}$ & $\begin{array}{l}\mathrm{DC} \\
(9) \\
\end{array}$ & $\begin{array}{l}Y^{a} O^{a} \\
(10) \\
\end{array}$ & $\begin{array}{c}\text { Reference }^{\mathrm{b}} \\
(11) \\
\end{array}$ \\
\hline 1 & G79. $34+0.33$ & 203227.0 & +40 & 21 & 06.5 & 4.23 & 4.01 & 38.29 & 088 & 0.70 & $\mathrm{I}^{E(1)}, \mathrm{M}^{I(1), F(1)}$ & (1) \\
\hline 2 & $\mathrm{DF}+25.90-0.17$ & 183909.4 & -06 & 20 & 07.3 & 0.79 & 0.43 & 05.64 & 108 & 0.19 & & (2) \\
\hline 3 & $\mathrm{DF}+30.31-0.28$ & 184738.7 & -02 & 27 & 57.6 & 0.93 & 0.58 & 07.34 & 004 & 0.25 & $\mathrm{~S}^{F(2)}$ & (2) \\
\hline 4 & $\mathrm{DF}+30.36+0.11$ & 184620.5 & -02 & 14 & 06.8 & 0.67 & 0.20 & 03.47 & 039 & 0.10 & & (2) \\
\hline 5 & $\mathrm{DF}+36.95+0.22$ & 185759.1 & +03 & 41 & 46.6 & 4.45 & 4.27 & 29.45 & 109 & 0.29 & $\mathrm{~S}^{I(1)}$ & (2) \\
\hline 6 & $\mathrm{DF}+51.47+0.00(\mathrm{~B})$ & 192612.1 & +16 & 25 & 49.0 & 2.29 & 1.81 & 14.39 & 091 & 0.38 & $\mathrm{~S}^{F(1), I I(5)}$ & (2) \\
\hline 7 & G305.136+0.068 & 131040.7 & -62 & 43 & 12.6 & 0.64 & 0.42 & 05.19 & 060 & 0.36 & $\mathrm{~S}^{I(1)}$ & (3) \\
\hline 8 & G333.125-0.562 & 162134.5 & -50 & 40 & 59.7 & 0.44 & 0.30 & 02.95 & 107 & 0.05 & $\mathrm{H}(1)$ & (3) \\
\hline 9 & $18090-1832-2$ & 181200.9 & -18 & 31 & 19.9 & 0.48 & 0.13 & 02.00 & 069 & 0.06 & & (4) \\
\hline 10 & $18151-1208-2$ & 181750.2 & -12 & 07 & 54.1 & 0.58 & 0.43 & 04.84 & 011 & 0.16 & $\mathrm{~S}^{I(2), I I(}$ & (4) \\
\hline 11 & $18247-1147-3$ & 182733.8 & -11 & 44 & 23.2 & 1.30 & 0.36 & 06.09 & 038 & 0.13 & $\cdots$ & (4) \\
\hline 12 & $18308-0841-2$ & 183334.3 & -08 & 38 & 38.7 & 0.40 & 0.29 & 03.33 & 324 & 0.15 & $\ldots$ & (4) \\
\hline 13 & $18308-0841-3$ & 183329.2 & -08 & 38 & 14.7 & 0.58 & 0.27 & 03.97 & 074 & 0.16 & $\cdots$ & (4) \\
\hline 14 & $18308-0841-5$ & 183334.4 & -08 & 37 & 37.5 & 0.36 & 0.14 & 02.01 & 342 & 0.04 & $\cdots$ & (4) \\
\hline 15 & $18348-0616-2 c$ & 183727.9 & -06 & 14 & 08.4 & 0.45 & 0.33 & 03.79 & 029 & 0.24 & $\cdots$ & (4) \\
\hline 16 & $18385-0512-3$ & $1841 \quad 17.3$ & -05 & 10 & 04.3 & 0.68 & 0.53 & 05.95 & 009 & 0.38 & $\ldots$ & (4) \\
\hline 17 & $18431-0312-3$ & 184545.1 & -03 & 08 & 41.0 & 0.56 & 0.24 & 03.25 & 110 & 0.12 & & (4) \\
\hline 18 & $18431-0312-4$ & 184553.3 & -03 & 08 & 48.8 & 1.08 & 0.75 & 08.64 & 346 & 0.12 & $\cdots$ & (4) \\
\hline \multicolumn{13}{|c|}{ This is a cut-in data } \\
\hline 71 & G33.36-0.01 & 185214.4 & +00 & 22 & 36.1 & 1.95 & 0.97 & 12.32 & 355 & 0.29 & $\mathrm{~S}^{I(1), I I(4)}$ & (6) \\
\hline 72 & $\mathrm{G} 33.42+0.13$ & 185152.9 & +00 & 29 & 33.0 & 0.57 & 0.31 & 04.15 & 004 & 0.29 & & (6) \\
\hline 73 & $\mathrm{G} 34.26+0.19$ & 185312.7 & +01 & 15 & 53.2 & 1.67 & 0.58 & 10.06 & 079 & 0.27 & $\mathrm{~S}^{I(1), I I(4)}$ & (6) \\
\hline 74 & G34.74+0.01 & 185444.2 & +01 & 36 & 58.5 & 0.87 & 0.54 & 06.36 & 028 & 0.27 & $\cdots$ & (6) \\
\hline 75 & G35.04-0.47 & 185658.8 & +01 & 39 & 50.5 & 0.59 & 0.44 & 05.39 & 326 & 0.29 & $\mathrm{~S}^{F(1), I I(2)}$ & (6) \\
\hline 76 & G34.63-1.03 & 185818.8 & +01 & 01 & 04.5 & 3.02 & 1.79 & 12.39 & 086 & 0.59 & $\mathrm{~S}^{F(1)}$ & (6) \\
\hline 77 & G35.02-1.50 & 190034.7 & +01 & 10 & 39.3 & 1.41 & 1.07 & 11.09 & 128 & 0.46 & $\ldots$ & (6) \\
\hline 78 & G43.19-0.16 & 191053.2 & +09 & 02 & 30.1 & 0.42 & 0.21 & 02.93 & 004 & 0.31 & & (6) \\
\hline 79 & $\mathrm{G} 43.32-0.20$ & 191117.5 & +09 & 08 & 19.7 & 1.01 & 1.00 & 06.55 & 099 & 0.33 & $\mathrm{~S}^{I(1)}$ & (6) \\
\hline 80 & $\mathrm{G} 43.78+0.05$ & 191113.6 & +09 & 39 & 39.9 & 1.32 & 0.96 & 08.55 & 011 & 0.32 & $\ldots$ & (6) \\
\hline 81 & G43.64-0.82 & 191408.2 & +09 & 05 & 57.3 & 2.85 & 2.51 & 08.62 & 002 & 0.53 & $\ldots$ & (6) \\
\hline 82 & $\mathrm{G} 48.84+0.15$ & 192029.9 & +14 & 10 & 55.3 & 1.22 & 0.61 & 06.22 & 001 & 0.31 & $\mathrm{~S}^{F(2)}$ & (6) \\
\hline 83 & $\mathrm{G} 48.84+0.14$ & 192034.6 & +14 & 11 & 36.2 & 1.05 & 0.66 & 07.04 & 005 & 0.27 & $\mathrm{~S}^{I(1)}$ & (6) \\
\hline 84 & $\mathrm{G} 50.07+0.06$ & 192314.0 & +15 & 13 & 49.0 & 0.81 & 0.47 & 06.08 & 022 & 0.47 & $\mathrm{~S}^{I(2)}$ & (6) \\
\hline 85 & G51.00-0.18 & 192254.1 & +15 & 56 & 11.1 & 2.57 & 1.91 & 15.13 & 353 & 0.40 & $\mathrm{I}^{E(1), P(1)}$ & (6) \\
\hline 86 & G53.88-0.18 & 193142.6 & +18 & 28 & 00.5 & 0.74 & 0.43 & 04.21 & 344 & 0.40 & $\ldots$ & (6) \\
\hline 87 & $\mathrm{G} 61.52+0.02$ & 194708.1 & +25 & 12 & 38.7 & 1.82 & 1.80 & 09.32 & 319 & 0.52 & $\mathrm{~S}^{I I(1)}$ & (6) \\
\hline
\end{tabular}

NOTE. - The full version of this table is available in electronic form. This catalog contains 87 IRDC cores selected from many literatures. All the information in columns is the same as those in the table 2 except that this has one more column as the last one where references for the sources are given.

${ }^{\mathrm{b}}$ References; (1) Carey et al. (1998), (2) Teyssier et al. (2002), (3) Garay et al. (2004), (4) Sridharan et al. (2005), (5) Rathborne et al. (2006), and (6) Ragan et al. (2006). 
TABLE 4

THE SJRC CORES.

\begin{tabular}{|c|c|c|c|c|c|c|c|c|c|c|c|}
\hline $\begin{array}{l}\text { Num. } \\
\text { (1) }\end{array}$ & $\begin{array}{l}\text { Source } \\
\text { (2) }\end{array}$ & $\begin{array}{l}\text { R.A. } \\
(2000) \\
(3)\end{array}$ & & $\begin{array}{c}\text { Dec. } \\
(2000 \\
(4)\end{array}$ & & $\begin{array}{c}\mathrm{a} \\
\left({ }^{\prime}\right) \\
(5)\end{array}$ & $\begin{array}{c}\mathrm{b} \\
\left({ }^{\prime}\right) \\
(6)\end{array}$ & $\begin{array}{l}\text { ED } \\
\left({ }^{\prime}\right) \\
(7)\end{array}$ & $\begin{array}{l}\mathrm{PA} \\
\left({ }^{\circ}\right) \\
(8)\end{array}$ & $\begin{array}{l}\text { DC } \\
(9)\end{array}$ & $\begin{array}{l}Y^{a}{ }^{a} \\
(10)\end{array}$ \\
\hline 1 & G000.00+00.65 & 174304.5 & -28 & 33 & 41.2 & 0.9 & 0.8 & 00.54 & 125 & 0.08 & \\
\hline 2 & G000.00-00.59 & 174758.4 & -29 & 14 & 57.9 & 1.1 & 0.7 & 00.62 & 125 & 0.06 & \\
\hline 3 & G000.00-00.74 & $1748 \quad 32.2$ & -29 & 9 19 & 15.6 & 1.3 & 0.6 & 00.61 & 095 & 0.07 & .. \\
\hline 4 & G000.00-00.33 & 174653.2 & -29 & 905 & 42.7 & 1.3 & 1.0 & 01.00 & 099 & 0.13 & \\
\hline 5 & G000.00-00.47 & 174728.8 & -29 & 11 & 03.3 & 1.6 & 0.7 & 00.87 & 146 & 0.15 & $\mathrm{~S}^{F(4),}$ \\
\hline 6 & G000.00-00.98 & 174931.7 & -29 & 27 & 02.7 & 0.8 & 0.4 & 00.28 & 215 & 0.04 & $\cdots$ \\
\hline 7 & G000.00-00.78 & 174842.8 & -29 & 20 & 18.2 & 0.9 & 0.8 & 00.53 & 218 & 0.07 & \\
\hline 8 & $\mathrm{G} 000.01+00.63$ & 174311.9 & -28 & 335 & 20.9 & 1.2 & 1.1 & 01.09 & 123 & 0.10 & . \\
\hline 9 & G000.02+00.57 & 174325.6 & -28 & 336 & 30.4 & 2.2 & 0.8 & 01.38 & 104 & 0.16 & \\
\hline 10 & G000.02-00.98 & 174931.5 & -29 & 25 & 28.8 & 1.3 & 0.7 & 00.70 & 235 & 0.11 & \\
\hline 11 & G000.03-00.35 & 174704.5 & -29 & 905 & 27.3 & 1.1 & 1.1 & 00.94 & 245 & 0.09 & $\mathrm{~S}^{I I(1)}$ \\
\hline 12 & G000.04-00.64 & $1748 \quad 16.5$ & -29 & 913 & 33.2 & 1.3 & 0.6 & 00.63 & 117 & 0.06 & $\mathrm{~S}^{F(1), I I(}$ \\
\hline 13 & G000.05-00.55 & 174755.7 & -29 & 10 & 36.6 & 1.3 & 0.8 & 00.87 & 241 & 0.09 & \\
\hline 14 & G000.05-00.68 & 174828.7 & -29 & 14 & 56.9 & 1.1 & 0.8 & 00.71 & 125 & 0.15 & $\mathrm{~S}^{I I(2)}$ \\
\hline 15 & G000.05-00.68 & 174832.7 & -29 & 914 & 04.5 & 1.9 & 0.9 & 01.38 & 095 & 0.21 & $\mathrm{~S}^{I(1), I I(3)}$ \\
\hline 16 & $\mathrm{G} 000.06+00.21$ & 174455.4 & -28 & 346 & 38.2 & 1.1 & 0.8 & 00.70 & 246 & 0.11 & $\mathrm{~S}^{I I(3)}$ \\
\hline 17 & G000.06-00.77 & 174855.3 & -29 & 15 & 17.6 & 1.5 & 0.9 & 01.08 & 217 & 0.18 & - \\
\hline 18 & G000.06-00.77 & 174849.1 & -29 & 16 & 22.4 & 1.5 & 1.0 & 01.17 & 260 & 0.17 & \\
\hline \multicolumn{12}{|c|}{ This is a cut-in data } \\
\hline 12756 & G359.91 & 1742 & -28 & 341 & 1 & 0.9 & 0.7 & 00.49 & 215 & 0.05 & - \\
\hline 12757 & G359.91+00.73 & 174235.1 & -28 & 37 & 33.3 & 1.1 & 0.7 & 00.63 & 116 & 0.07 & \\
\hline 12758 & G359.91+00.17 & 174447.7 & -28 & 35 & 53.8 & 1.6 & 1.3 & 01.59 & 251 & 0.64 & 3) \\
\hline 12759 & G359. $91+00.17$ & 174443.6 & -28 & 35 & 27.8 & 1.3 & 1.1 & 01.20 & 131 & 0.37 & $\mathrm{~S}^{I(3), F(3), I I(25)}$ \\
\hline 12760 & G359.91+00.39 & 174352.4 & -28 & 47 & 48.2 & 1.6 & 0.8 & 00.96 & 125 & 0.11 & $\mathrm{~S}^{I I(1)}$ \\
\hline 12761 & G359.92-00.86 & 174850.3 & -29 & 26 & 53.3 & 1.2 & 0.9 & 00.86 & 145 & 0.10 & .. \\
\hline 12762 & G359.93+00.61 & 174303.5 & -28 & 340 & 08.4 & 1.1 & 1.0 & 00.86 & 162 & 0.07 & \\
\hline 12763 & G359.94-00.34 & 174652.3 & -29 & 909 & 44.0 & 0.9 & 0.7 & 00.53 & 117 & 0.0 & $\mathrm{~S}^{I I(2)}$ \\
\hline 12764 & G359.94+01.20 & 174047.4 & -28 & 320 & 51.4 & 0.9 & 0.8 & 00.56 & 251 & 0.09 & \\
\hline 12765 & G359.95+00.59 & 174312.3 & -28 & 39 & 46.4 & 1.2 & 0.9 & 00.82 & 126 & 0.08 & $\mathrm{~S}^{I I(1)}$ \\
\hline 12766 & G359.95-00.94 & 174913.9 & -29 & 27 & 39.8 & 1.1 & 0.8 & 00.67 & 125 & 0.09 & $\mathrm{~S}^{I I(1)}$ \\
\hline 12767 & G359.96-00.76 & 174832.4 & -29 & 22 & 18.4 & 1.0 & 0.7 & 00.59 & 091 & 0.06 & $\cdots$ \\
\hline 12768 & G359.96+01. 15 & 174103.8 & -28 & 321 & 32.9 & 0.7 & 0.6 & 00.34 & 125 & 0.07 & $\cdots$ \\
\hline 12769 & G359.96-00.78 & 174837.1 & -29 & 922 & 08.6 & 1.2 & 1.0 & 00.92 & 099 & 0.09 & . \\
\hline 12770 & G359.98-00.81 & 174847.5 & -29 & 22 & 11.2 & 0.9 & 0.6 & 00.45 & 143 & 0.04 & $\cdots$ \\
\hline 12771 & G359.98-01.01 & 174932.0 & -29 & 928 & 41.4 & 0.9 & 0.9 & 00.66 & 191 & 0.10 & .. \\
\hline 12772 & G359.98+00.27 & 174431.7 & -28 & 34 & 40.1 & 0.9 & 0.7 & 00.50 & 260 & 0.05 & $\mathrm{~S}^{I I(2)}$ \\
\hline 12773 & G359.99+00.50 & 174339.4 & -28 & 340 & 58.7 & 1.3 & 0.7 & 00.72 & 215 & 0.06 & $\ldots$ \\
\hline 12774 & G359.99-01.21 & 175023.3 & -29 & 34 & 19.3 & 1.2 & 0.6 & 00.63 & 260 & 0.14 & $\cdots$ \\
\hline
\end{tabular}

NoTE.-The full version of this table is available in electronic form. This catalog contains the 12,774 SJRC IRDC cores. All the information in columns is the same as those in the table 2 . 


\section{(c) Summary of the IRDC Cores Collected}

In total, 13,650 IRDC cores were collected for this study. These consist of 12,774 IRDC cores from SJRC catalog, 87 IRDC cores from various studies mentioned above, and 789 IRDC cores newly selected from this study. All these IRDC cores are listed in Table 2 for 789 IRDC cores, Table 3 for 87 IRDC cores, and Table 4 for the SJRC catalog with some useful information such as their geometric properties and YSOs association of the IRDC cores which will be discussed in next section $\|$.

In the tables the sequential numbers of the IRDC cores are given in column 1 , the source name in column 2, the R.A. and Dec. [J2000] of the IRDC cores in column 3 and 4 , the diameter of the major and minor axes of the IRDC cores in column 5 and 6 , the Equivalent Diameter (ED) of the IRDC cores in column 7, the Position Angle (PA) in column 8, the Darkness Contrast (DC) of the IRDC cores in column 9, possible YSOs association in column 10. Note that table 3 has column 11 where references are given.

\section{ASSOCIATION OF THE IRDC CORES WITH YSOS}

Whether IRDC cores are associated with newly born "young" stars is an important issue for understanding how stars form in the IRDC cores. In this section we search for YSO candidates using various criteria, explore the association possibility of IRDC cores with YSOs and discuss implication of the statistics of starless or starred IRDC cores in high-mass star formation. Here YSOs refer to protostars which correspond to class 0 or I (sometimes called as embedded YSO-EYSO) and PMS stars (Flat spectrum, class II or III).

\section{(a) Identification of YSO Candidates}

The IRAS point sources meeting the criteria suggested by Lee \& Myers (1999), mainly rising flux densities at the longer IRAS bands, were used to search for EYSO candidates lying within the IRDC cores in the range of $0^{\circ}<l<360^{\circ}$ and $|b|<3^{\circ}$. However, the IRAS sensitivity restricts detection to sources stronger than $\sim 0.1 \mathrm{~L}_{\odot}$ at the Taurus distance $(\sim 140 \mathrm{pc})$ (Myers et al. 1987); EYSOs would not be detected in the mostly distant (over a few kpc) IRDC cores unless they are at least as bright as $\sim 80 \mathrm{~L}_{\odot}$ at $4 \mathrm{kpc}$. We found only 172 IRDC cores from the search of entire IRDC cores to contain IRAS point sources. We also used color-color diagram criteria in 12,25 , and $60 \mu \mathrm{m}$ IRAS bands of Weintraub et al. (1990) to find a possible PMS star within an IRDC core; $-2.00<\frac{\log \left(\nu_{12} \mathrm{~F}_{12} / \nu_{25} \mathrm{~F}_{25}\right)}{\log \left(\nu_{12} / \nu_{25}\right)}<1.35$ and $-1.75<\frac{\log \left(\nu_{25} \mathrm{~F}_{25} / \nu_{60} \mathrm{~F}_{60}\right)}{\log \left(\nu_{25} / \nu_{60}\right)}<2.20$. Fifty eight

\footnotetext{
"Here we present the part of the tables only. The full version of the tables is available in electronic form at http://gjkim.kasi.re.kr/IRDC.
}

IRDC cores were found to have possible PMS stars.

The MSX survey also provides a catalog of nearly 0.45 million point sources (MSX point source catalog v2.3 from NASA/IRSA Archive) with detection sensitivity of $100-10,000 \mathrm{mJy}$ at $4.29,4.35,8.28,12.13$, 14.65 , and $21.34 \mu \mathrm{m}$ within the region of $|1|<180^{\circ}$ and $|\mathrm{b}|<5^{\circ}$ (Egan et al. 1999). A total of 18,220 point sources are possibly YSOs according to the slope of the SED from 4.29 to $21.34 \mu \mathrm{m}$. Sixty nine IRDC cores are found to coincide with the positions of some of these YSO candidates.

Recent SST missions explore very faint embedded sources in dense molecular cores (e.g., Young et al. 2004; Bourke et al. 2006; Dunham et al. 2006; Lee et al. 2009). In particular, the GLIMPSE legacy program conducted a survey of the inner galaxy at 3.6, $4.5,5.8$, and $8.0 \mu \mathrm{m}$, covering the region of $|1|<65^{\circ}$ and $|\mathrm{b}|<0.75^{\circ} \sim 4.2^{\circ}$ and released a point source catalog** of nearly 70 million from GLIMPSE survey. The GLIMPSE point source catalog lists highly reliable point sources that have detection at least twice in one IRAC band and at least once in an adjacent band, using the high angular resolution $\left(\leq 2^{\prime \prime}\right)$ and high sensitivity $(1 \sigma \sim 0.2-0.4 \mathrm{mJy})$ IRAC (InfraRed Array Camera; Fazio et al. 2004). GLIMPSE point sources were identified as EYSO or PMS candidates, and assigned to one of four classes based on the slope $(\alpha)$ of the Spectral Energy Distribution (SED) from 3.6 to 8.0 $\mu \mathrm{m}\left(\alpha=\frac{\operatorname{dlog} \lambda f_{\lambda}}{\operatorname{dlog} \lambda}\left[\mathrm{Wm}^{-2}\right]\right)$. Following the definition by Greene et al. (1994), we make classifications as class I $(\alpha \geq 0.3)$, Flat spectrum $(-0.3 \leq \alpha<0.3)$, class II $(-$ $1.6 \leq \alpha<-0.3)$, and class III $(\alpha<-1.6)$. A histogram of the observed values of $\alpha$ is shown in Figure 4.

For class III objects it is not easy to distinguish them from main sequence stars without complementary data such as X-ray fluxes. So the identification of class III is not specifically given in this paper. In the classification we minimized contamination from unrelated sources by dropping sources fainter than $\mathrm{m}_{3.6}=14^{\mathrm{m}}$ to eliminate possible faint high red-shift AGNs (Allen et al. 2007). The GLIMPSE survey region contains about 198,045 point sources which may be EYSOs (class I objects) or PMSs (Flat spectrum objects or class II objects). The mean surface density of the GLIMPSE point sources is about 0.13 sources per arc $\min ^{-2}$. The typical angular size of the IRDC cores within GLIMPSE survey region is about $1^{\prime}$. Hence, the likelihood that a line-ofsight coincidence of the point sources with the IRDC cores is due to chance alignment of a foreground or background source would be about 0.13 point sources per IRDC core, implying that the alignment of a foreground or background source with an IRDC core is relatively unlikely. Thus, the assumption that GLIMPSE point sources satisfying the redness criterion and align-

** This point source catalogs consist of GLIMPSE I spring '07, GLIMPSE II spring '08 and GLIMPSE 3D (2007-2009). See http : //data.spitzer.caltech.edu/popular/GLIMPSE/ 
ing with the IRDC core are associated to the IRDC core is reasonable. A total of 12,200 IRDC cores within the GLIMPSE survey area were examined to see if the GLIMPSE point sources (i.e., EYSO or PMS star candidates) were located within the FWHMin contours of the IRDC cores. Of the 12,200 IRDC cores, 4,028 were found to have at least one GLIMPSE point source.

An association of maser sources with the IRDC cores was also checked. Masers are known to be a good indicator of the early stages of star formation and may also be a good tracer of star-forming activities. We searched for the presence of masers within the IRDC cores using the Arcetri Catalog of water masers (Valdettaro et al. 2001) and the General Catalog of $6.7 \mathrm{GHz}$ methanol masers (Pestalozzi \& Minier 2003) finding that 8 and 33 IRDC cores include water and methanol masers, respectively.

\section{(b) Association Check of the IRDC Cores with YSO Candidates}

We assumed that IRDC cores might have association with the YSO candidates if the YSOs are positioned within the FWHMin contour of the IRDC core. However, it is practically very difficult to check every association of YSOs for all IRDC cores by eye. Therefore we made an ellipse fitting to the FWHMin contour of each IRDC and compared the position of the YSO with dimension of the fitted ellipse. If YSOs are located within the radius of the minor axis of the ellipse, these are assumed to be associated with IRDC cores. On the other hand if YSOs are located outside the radius of the major axis of the ellipse, these are regarded not to be associated with the IRDC core. The YSOs that are located between the radii of the minor and major axes of the ellipse were directly checked with eyes to see if they are located within the area of the ellipse or not.

\section{(c) Statistics of the IRDC Cores with or with- out (embedded) YSO Candidates, and its Implication and Limitation}

In this way we found $4,036,69,172,8$, and 33 IRDC cores containing GLIMPSE, MSX, IRAS point sources, water maser and methanol maser sources within the ellipse of the IRDC core, respectively (Table 1). We note that 33 IRDC cores with the methanol maser have the association by other YSO candidates, too.

Therefore 9,540 out of 13,650 IRDC cores have no YSO candidates while 4,110 IRDC cores are possibly associated with YSO candidates. Dealing with GLIMPSE point sources and IRDC cores within the GLIMPSE area, we found that 8,102 IRDC cores have no YSO association while 4,028 IRDC cores have YSOs.

Out of 13,650 IRDC cores, 1,077 cores are found to have EYSO candidates and thus this ratio of N(IRDC core with protostars)/N(starless IRDC core) is about $0.11(\sim 1,077 / 9,540)$. Within the GLIMPSE survey area, there are 1,072 IRDC cores with protostars and thus the ratio of $\mathrm{N}$ (IRDC core with protostars)/N(starless IRDC core $)$ is about $0.13(\sim 1,072 / 8,102)$, which is much less than that of low-mass cores (about 0.31; Lee \& Myers 1999). Recently Peretto and Fuller (2009) have examined the association of IRDC cores with 24 $\mu \mathrm{m}$ sources from Multiband Imaging Photometer for Spitzer GALactic plane survey (MIPSGAL), suggesting that about $20-68 \%$ of the IRDC cores show the association with $24 \mu \mathrm{m}$ sources. Parsons et al. (2009) also suggested that two-thirds of the cores detected at $850 \mu \mathrm{m}$ are associated with $24 \mu \mathrm{m}$ sources. Our fractional ratio of the IRDC cores with embedded YSOs is therefore smaller than these values. However, M. Kim et al. (in prep) have found that a significant fraction (over $30 \%$ ) of $24 \mu \mathrm{m}$ sources are either galaxies or AGB stars instead of being EYSOs. Thus it is possible that their estimation of the fraction of the IRDC cores with EYSOs is overestimated in the sensitivity limited sample. The lifetime range of embedded high-mass stars is suggested to be between $10^{3} \sim 10^{4}$ years (Wood \& Churchwell 1989a,b; Van der walt 2005; Motte et al. 2007; Pestalozzi et al. 2007). Therefore, if all the IRDC cores are in a pathway to the high-mass star formation, the fractional ratio of the IRDC cores with EYSOs may imply that the IRDC cores spend about $10^{4} \sim 10^{5}$ years to form high-mass stars.

However, it should be noted that this ratio for the IRDC cores can be much more uncertain than the ratio for the low-mass cores. The Spitzer legacy Cores to Disks (c2d) program (Evans et al. 2003) has surveyed 67 low-mass starless cores and found Very Low Luminosity Objects (VeLLOs) in $\sim 20 \%$ of the sample (Dunham et al. 2008), which would only slightly affect the ratio of the starred cores to starless cores. However, the ratio for the IRDC core can be significantly affected because the IRDC cores are usually much more distant than the nearby low-mass cores, and all the surveys are sensitivity-limited.

We calculated the luminosity of the faintest protostar in the IRDC cores that GLIMPSE survey has detected. In the calculation we assumed that the protostar has the same Spectral Energy Distribution as assumed by Myers et al. (1987). We also assumed that the observed peak flux density of the protostar would be the maximum over the entire spectrum and the protostar had the spectrum of a blackbody for wavelengths greater than or equal to the wavelength of the peak flux density. The minimum flux density $(\sim 0.36 \mathrm{mJy})$ at $8.0 \mu \mathrm{m}$ among the source detected at all 4 bands in the GLIMPSE survey was compared with the minimum flux density that IRAS would detect at the same wavelength as inferred from an extrapolation of the model flux density to $8 \mu \mathrm{m}$. The minimum detectable luminosity of GLIMPSE point sources was calculated to be about $1.17\left(\frac{4}{\mathrm{~d} \mathrm{kpc}}\right)^{2} \mathrm{~L}_{\odot}$, where $\mathrm{d}$ is a distance of the IRDC core from us. This limit is much higher than the minimum detectable luminosity $(\sim 0.1 \mathrm{~L} \odot)$ characteristic of the VeLLOs in the low-mass cores. Hence, the ratio for the IRDC cores can be larger than the given 
values if the observing sensitivity of GLIMPSE were better.

\section{STATISTICS OF THE IRDC CORES}

Using geometric properties of all collected 13,650 IRDC cores and their association with YSOs, we discuss the statistics of various parameters of the IRDC cores.

\section{(a) Multiplicity of YSOs in the IRDC Cores}

Figure 5 shows a number distribution of 13,650 IRDC cores according to the number of YSO candidates. The IRDC cores without YSO candidate occupy about $70 \%$ of the entire IRDC cores while the IRDC cores with YSO candidates do about $30 \%$. It is interesting to note that about $49 \%$ of the starred IRDC cores have a single YSO candidate while others ( $\sim 51 \%$ ) have multiple YSOs. Considering that even a GLIMPSE point source found as a single with SST having poor spatial resolution can be possibly double or multiple stars, this significant fraction of the IRDC cores with multiple YSOs may suggest that many IRDC cores are likely multiple star formation sites.

\section{(b) $\sqrt{a b}$ and $\mathbf{a} / \mathbf{b}$ of the IRDC Cores}

The number distribution of the apparent geometric angular size $(\sqrt{a b})$ of the IRDC core is presented in Figure 6 . Most of 13,650 IRDC cores $(\sim 76 \%)$ have angular sizes between $0^{\prime} .7$ and $1^{\prime} .2$ with a mean ( \pm s.e.m.) of $1^{\prime} .012 \pm 0^{\prime} .002$. Figure 6 also shows that the angular size of the IRDC cores is somewhat dependent upon how the IRDC cores contain YSOs. The starless IRDC cores have a mean angular size of $0^{\prime} .965 \pm 0^{\prime} .002$ while the starred IRDC cores have that of $1^{\prime} .12 \pm 0^{\prime} .01$. The mean angular size of IRDC cores with a single YSO and two or more YSO candidates are $1^{\prime} .05 \pm 0^{\prime} .01$ and $1^{\prime} .19 \pm 0^{\prime} .01$, respectively. This indicates that the angular size of IRDC cores has a slight tendency to be larger as the IRDC cores have more YSOs, although the difference among them is small compared to their angular sizes.

Figure 7 displays the number distribution of the aspect ratio $(\mathrm{a} / \mathrm{b})$ of all IRDC cores, and the IRDC cores with and without YSOs. The aspect ratio of all IRDC cores is mostly $(\sim 80 \%)$ between 1.0 and 1.9 with a mean of $1.61 \pm 0.01$. The aspect ratios for the IRDC cores with and without YSO candidates are $1.82 \pm 0.01$ and $1.51 \pm 0.01$, respectively. The mean aspect ratio of IRDC cores with a single YSO candidate is $1.68 \pm 0.01$ while that of IRDC cores with two or more YSO candidates is $1.96 \pm 0.02$. These statistics are also compared with the mean aspect ratios of low-mass cores without and with YSO candidates, $2.4 \pm 0.1$ and $2.2 \pm 0.2$, respectively (Lee \& Myers 1999). Therefore the IRDC cores with more YSOs tend to be more elongated, but less elongated than the low-mass cores.

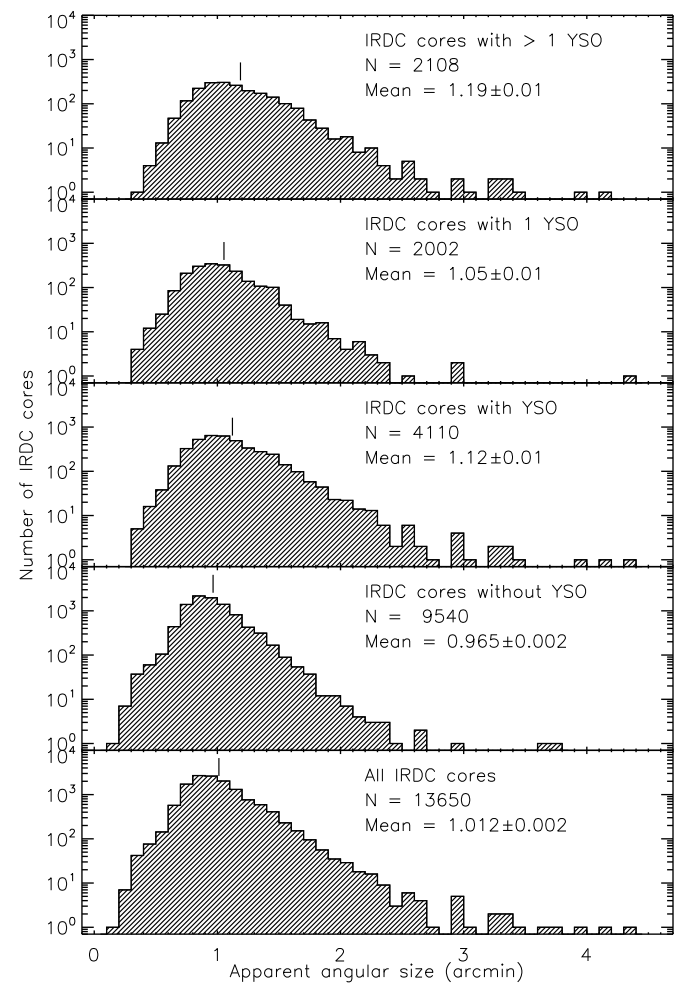

Fig. 6. - A number distribution of the apparent angular size of the IRDC with its geometric $\sqrt{a b}$. Most of 13,650 IRDC cores $(\sim 76 \%)$ have angular sizes between $0^{\prime} .7$ and $1^{\prime} .2$ and a mean angular size of $1^{\prime} .012 \pm 0^{\prime} .002$. The positions of mean angular sizes for the IRDC cores in several groups are marked with vertical bars in each panel. The figure also shows that the angular size of the IRDC cores with YSO, are slightly larger than the starless IRDC cores.

\section{(c) Darkness Contrast of the IRDC Cores with and Without YSO}

The darkness contrast may represent the column density toward IRDC cores and thus it may be interesting to investigate the distribution of darkness contrast for two sub-groups of IRDCs with and without YSOs. A natural prediction would be that the IRDC cores having higher column density will have more chance to have YSOs and thus the IRDC cores with YSOs have better darkness contrast than IRDC cores without YSOs.

Figure 8 shows the number distribution of the darkness contrast for two sub-groups of starred IRDCs and starless IRDCs for two samples of SJRC IRDCs and other (789 and 87) IRDC cores that we selected. (We investigated the distribution of the darkness contrast for two samples because the definition of the background level of the brightness is somewhat different in two samples). In the sample of SJRC IRDC cores, the starless IRDC cores have a mean darkness contrast of $0.221 \pm 0.001$ while the starred IRDC cores have that of $0.251 \pm 0.001$. In other sample, the starless IRDC cores 


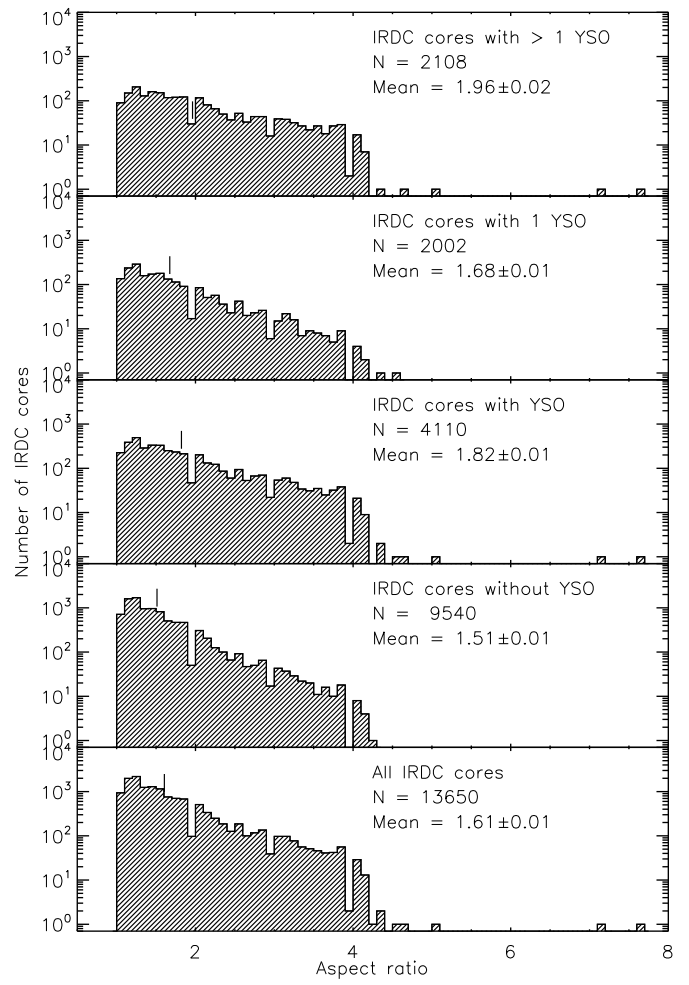

Fig. 7.- A number distribution of the aspect ratio (a/b) of IRDC cores. The positions of mean aspect ratios for the IRDC cores in several groups are marked with vertical bars in each panel. The aspect ratio of all IRDC cores is mostly $(\sim 80 \%)$ between 1.0 and 1.9 with a mean of $1.61 \pm 0.01$. The figure also shows that the aspect ratio of the IRDC cores with YSO, are slightly larger than the starless IRDC cores.

have a mean darkness contrast of $0.265 \pm 0.005$ while the starred IRDC cores have that of $0.292 \pm 0.005$. It is interesting to note that in both samples the starred IRDC cores have higher darkness contrast than starless IRDC cores, indicating that the starred IRDC cores have higher column density than starless IRDC cores.

\section{SUMMARY}

Constructing a fully possible set of IRDC cores and examining an association of the IRDC cores with YSOs is the first step for a systematic study of their role in high-mass star formation. This paper examines the association of InfraRed Dark Cloud (IRDC) cores with YSOs and discusses properties of the IRDC cores. For this study a total of 13,650 IRDC cores were collected mainly from the catalogs of the IRDC cores published from other studies and partially from our subset of new 789 IRDC core candidates. Our new cores are mostly dark regions surrounded by bright PAH mid-infrared emissions of the background which are missed in previous other works of constructing catalogs of the IRDC cores. More than half of the sources that are larger

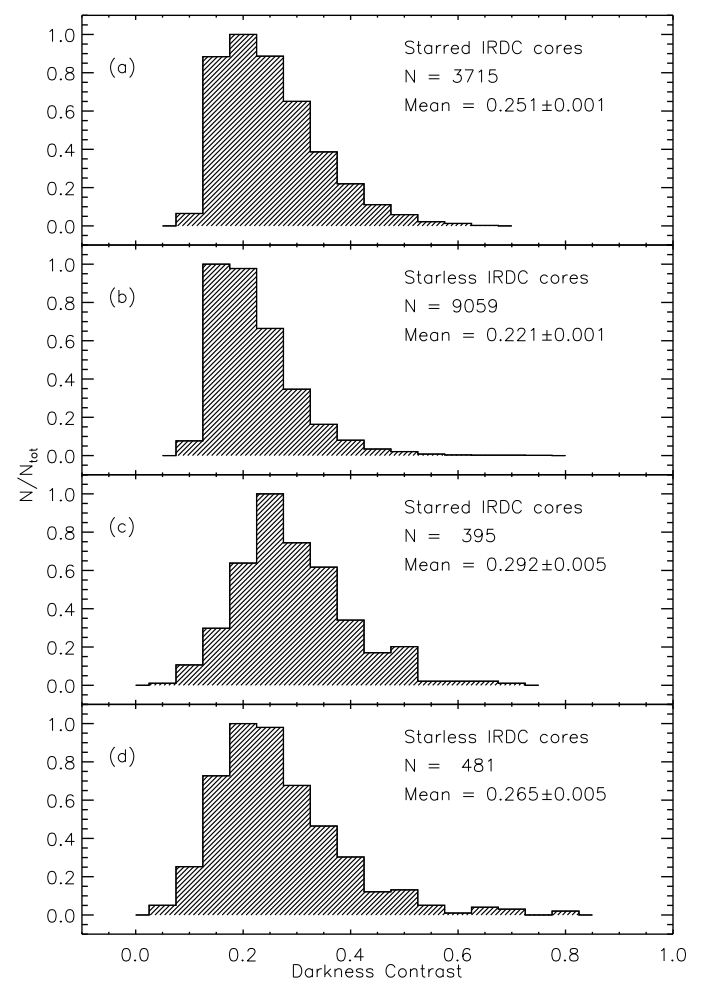

Fig. 8. - A number distribution of the darkness contrast of starred IRDC cores and starless IRDC cores. Upper two panels (a) and (b) are for the sample of SJRC IRDC cores and lower two panels (c) and (d) for IRDC cores that we collected for this study. In both samples the darkness contrast of starred IRDC cores tends to be higher than that of starless IRDC cores.

than $2^{\prime}$ are found to coincide with the distribution of ${ }^{13} \mathrm{CO}$ molecular emissions, implying that many of the selected IRDC cores are likely the real clouds.

The YSO candidates were identified with the slopes of the SED of the GLIMPSE, MSX, and IRAS point sources, and the existence of the water and methanol masers. The association of the IRDC cores with these YSOs was checked by their line-of-sight coincidence within the dimension of the IRDC core. Accounting for the duplicated association of multiple YSO indicators in the same IRDC core, a total of 4,110 IRDC cores are found to have YSO candidates.

Considering the 12,200 IRDC cores within the GLIMPSE survey region, for which the association of YSO candidates with IRDC cores is rather well examined, we found that 4,098 IRDC cores $(34 \%)$ have at least one YSO candidate and 1,072 IRDC cores among them have embedded YSOs, while the rest 8,102 (66\%) have no YSO candidate. The ratio of [N(IRDC core with protostars)]/[N(IRDC core without YSO)] for 12,200 IRDC cores is about 0.13 .

Taking into account this ratio and typical lifetime of high-mass embedded YSOs, we suggest that the 
lifetime of the starless IRDC cores would be about $10^{4} \sim 10^{5}$ years. However, we also found that the GLIMPSE point sources have a minimum detectable luminosity of about $1.2 \mathrm{~L} \odot$ at a typical IRDC distance of $\sim 4 \mathrm{kpc}$. Therefore, the fractional ratio of the IRDC cores with EYSOs given here should be a lower limit and the estimated lifetime of starless IRDC cores can be an upper limit.

It was also found that the geometric parameters of the IRDC cores vary depending on how many YSO candidates the IRDC cores contain. The IRDC cores with more YSOs tend to be larger, more elongated, and have better darkness contrast than the IRDC cores with fewer or no YSOs.

This research was supported by the Basic Research Program (KOSEF R01-2003-000-10513-0) of the Korea Science. J.B.P acknowledges support from UNAMPAPIIT grant 110606. This research has made use of the NASA/IPAC InfraRed Science Archive (MSX, IRAS, and Spitzer), which is operated by the Jet Propulsion Laboratory, California Institute of Technology, under contract with the National Aeronautics and Space Administration. This research also has made use of Arcetri Catalog of water masers (Valadettaro et al. 2001) and the General Catalog of $6.7 \mathrm{GHz}$ methanol masers (Pestalozzi \& Minnier 2003).

\section{REFERENCES}

Allen, L., Megeath, S. T., Gutermuth, R., Myers, P. C., Wolk, S., Adams, F. C., Muzerolle, J., Young, E., \& Pipher, J. L., 2007, The Structure and Evolution of Young Stellar Clusters, Protostars and Planets V, 361

Bally, J. \& Zinnecker, H., 2005, The Birth of High-Mass Stars: Accretion and/or Mergers?, AJ, 129, 2281

Benjamin, R. A., Churchwell, E., Babler, B. L., Bania, T. M., Clemens, D. P., Cohen, M., Dickey, J. M., Indebetouw, R., Jackson, J. M., Kobulnicky, H. A., Lazarian, A., Marston, A. P., Mathis, J. S., Meade, M. R., Seager, S., Stolovy, S. R., Watson, C., Whitney, B. A., Wolff, M. J., \& Wolfire, M. G. 2003, GLIMPSE. I. An SIRTF Legacy Project to Map the Inner Galaxy, PASP, 115, 953

Beuther, H., Sridharan, T. K., \& Saito, M., 2005, Caught in the Act: The Onset of Massive Star Formation, ApJ, 634, L185

Beuther, H., Churchwell, E. B., McKee, C. F., \& Tan, J. C., 2007, The Formation of Massive Stars, Protostars and Planets V, 165

Bourke, T. L., Myers, P. C., Evans, II, N. J., Dunham, M. M., Kauffmann, J., Shirley, Y. L., Crapsi, A., Young, C. H., Huard, T. L., Brooke, T. Y., Chapman, N., Cieza, L., Lee, C. W., Teuben, P., \& Wahhaj, Z. 2006, The Spitzer c2d Survey of Nearby Dense Cores. II. Discovery of a Low-Luminosity Object in the "Evolved Starless Core" L1521F, ApJ, 649, L37
Carey, S. J., Clark, F. O., Egan, M. P., Price, S. D. Shipman, R. F., \& Kuchar, T. A., 1998, The Physical Properties of the Midcourse Space Experiment Galactic Infrared-dark Clouds, ApJ, 508, 721

Carey, S. J., Feldman, P. A., Redman, R. O., Egan, M. P., MacLeod, J. M., \& Price, S. D., 2000, Submillimeter Observations of Midcourse Space Experiment Galactic Infrared-Dark Clouds, ApJ, 543, L157

Churchwell, E., 1990, Ultracompact H II regions - The impact of newly formed massive stars on their environment, A\&A Rev., 2, 79

Dunham, M. M., Evans, II, N. J., Bourke, T. L., Dullemond, C. P., Young, C. H., Brooke, T. Y., Chapman, N., Myers, P. C., Porras, A., Spiesman, W., Teuben, P. J., \& Wahhaj, Z., 2006, The Spitzer c2d Survey of Nearby Dense Cores. I. First Direct Detection of the Embedded Source in IRAM 04191+1522, ApJ, 651,945

Dunham, M. M., Crapsi, A., Evans, N. J., II, Bourke, T. L., Huard, T. L., Myers, P. C., \& Kauffmann, J., 2008, Identifying the Low-Luminosity Population of Embedded Protostars in the c2d Observations of Clouds and Cores, ApJS, 179, 249

Egan, M. P., Shipman, R. F., Price, S. D., Carey, S. J., Clark, F. O., \& Cohen, M., 1998, A Population of Cold Cores in the Galactic Plane, ApJ, 494, L199

Egan, M. P., Price, S. D., Shipman, R. F., Gugliotti, G. M., Tedesco, E. F., Moshir, M., \& Cohen, M., 1999, The MSX Infrared Point Source Catalog, Version 1. 0, ASPC , 177, 404

Evans, II, N. J., Allen, L. E., Blake, G. A., Boogert, A. C. A., Bourke, T., Harvey, P. M., Kessler, J. E., Koerner, D. W., Lee, C. W., Mundy, L. G., Myers, P. C., Padgett, D. L., Pontoppidan, K., Sargent, A. I., Stapelfeldt, K. R., van Dishoeck, E. F., Young, C. H., \& Young, K. E., 2003, From Molecular Cores to Planet-forming Disks: An SIRTF Legacy Program, PASP, 115, 965

Fazio, G. G., Hora, J. L. and Allen, L. E., Ashby, M. L. N., Barmby, P., Deutsch, L. K., Huang, J.-S., Kleiner, S., Marengo, M., Megeath, S. T., Melnick, G. J., Pahre, M. A., Patten, B. M., Polizotti, J., Smith, H. A., Taylor, R. S., Wang, Z., Willner, S. P., Hoffmann, W. F., Pipher, J. L., Forrest, W. J., McMurty, C. W., McCreight, C. R., McKelvey, M. E., McMurray, R. E., Koch, D. G., Moseley, S. H., Arendt, R. G., Mentzell, J. E., Marx, C. T., Losch, P., Mayman, P., Eichhorn, W., Krebs, D., Jhabvala, M., Gezari, D. Y., Fixsen, D. J., Flores, J., Shakoorzadeh, K., Jungo, R., Hakun, C., Workman, L., Karpati, G., Kichak, R., Whitley, R., Mann, S., Tollestrup, E. V., Eisenhardt, P., Stern, D., Gorjian, V., Bhattacharya, B., Carey, S., Nelson, B. O., Glaccum, W. J., Lacy, M., Lowrance, P. J., Laine, S., Reach, W. T., Stauffer, J. A., Surace, J. A., Wilson, G., Wright, E. L., Hoffman, A., Domingo, G. \& Cohen, M., 2004, The Infrared Array Camera (IRAC) for the Spitzer Space Telescope, ApJS, 154, 10 
Fiege, J. D., Johnstone, D., Redman, R. O., \& Feldman, P. A., 2004, A Genetic Algorithm-based Exploration of Three Filament Models: A Case for the Magnetic Support of the G11.11-0.12 Infrared-dark Cloud, ApJ, 616, 925

Greene, T. P., Wilking, B. A., Andre, P., Young, E. T., \& Lada, C. J., 1994, Further mid-infrared study of the rho Ophiuchi cloud young stellar population: Luminosities and masses of pre-main-sequence stars, ApJ, 434, 614

Garay, G., Faúndez, S., Mardones, D., Bronfman, L., Chini, R., \& Nyman, L.- $\AA$. 2004, Discovery of Four New Massive and Dense Cold Cores, ApJ, 610, 313

Hennebelle, P., Pérault, M., Teyssier, D., \& Ganesh, S., 2001, Infrared dark clouds from the ISOGAL survey. Constraints on the interstellar extinction curve, A\&A, 365, 598

Jackson, J. M., Rathborne, J. M., Shah, R. Y., Simon, R., Bania, T. M., Clemens, D. P., Chambers, E. T., Johnson, A. M., Dormody, M., Lavoie, R., \& Heyer, M. H., 2006, The Boston University-Five College Radio Astronomy Observatory Galactic Ring Survey, ApJS, 163, 145

Lee, C. W. \& Myers, P. C., 1999, A Catalog of Optically Selected Cores, ApJS, 123, 233

Lee, C. W., Bourke, T. L., Myers, P. C., Dunham, M., Evans, N., Lee, Y., Huard, T., Wu, J., Gutermuth, R., Kim, M.-R., \& Kang, H. W., 2009, The Spitzer c2d Survey of Nearby Dense Cores. V. Discovery of a VeLLO in the "Starless" Dense Core L328, ApJ, 693, 1290

Motte, F., Bontemps, S., Schilke, P., Schneider, N., Menten, K. M., \& Broguière, D., 2007, The earliest phases of high-mass star formation: a 3 square degree millimeter continuum mapping of Cygnus X, A\&A, 476, 1243

Myers, P. C., Fuller, G. A., Mathieu, R. D., Beichman, C. A., Benson, P. J., Schild, R. E., \& Emerson, J. P., 1987, Near-infrared and optical observations of IRAS sources in and near dense cores, ApJ, 319, 340

Norberg, P. \& Maeder, A., 2000, On the formation of massive stars by accretion, A\&A, 359, 1025

Ormel, C. W., Shipman, R. F., Ossenkopf, V., \& Helmich, F. P., 2005, The modelling of infrared dark cloud cores, A\&A, 439, 613

Parsons, H., Thompson, M. A., \& Chrysostomou, A., 2009, Infrared dark cloud cores in the SCUBA Legacy Catalogue, MNRAS, 399, 1506

Perault, M., Omont, A., Simon, G., Seguin, P., Ojha, D., Blommaert, J., Felli, M., Gilmore, G., Guglielmo, F., Habing, H., Price, S., Robin, A., de Batz, B., Cesarsky, C., Elbaz, D., Epchtein, N., Fouque, P., Guest, S., Levine, D., Pollock, A., Prusti, T., Siebenmorgen, R., Testi, L. \& Tiphene, D., 1996, First ISOCAM images of the Milky Way, A\&A, 315, L165
Peretto, N. \& Fuller, G. A., 2009, The initial conditions of stellar protocluster formation. I. A catalogue of Spitzer dark clouds, A\&A, 505, 405

Pestalozzi, M. R. \& Minier, V., 2003, General Catalogue of $6.7 \mathrm{GHz}$ Methanol Masers (Pestalozzi+, 2005), VizieR Online Data Catalog, 343, 20737

Pestalozzi, M. R., 2007, Methanol masers as tools to study high-mass star formation, IAU Symposium, 242,89

Pillai, T., Wyrowski, F., Carey, S. J., \& Menten, K. M., 2006, Ammonia in infrared dark clouds, A\&A, 450, 569

Price, S. D., 1995, Infrared Astronomy on the Midcourse Space Experiment, Space Science Reviews, 74,81

Ragan, S. E., Bergin, E. A., Plume, R., Gibson, D. L., Wilner, D. J., O'Brien, S., \& Hails, E., 2006, Molecular Line Observations of Infrared Dark Clouds: Seeking the Precursors to Intermediate and Massive Star Formation, ApJS, 166, 567

Rathborne, J. M., Jackson, J. M., Chambers, E. T., Simon, R., Shipman, R., \& Frieswijk, W., 2005, Massive Protostars in the Infrared Dark Cloud MSXDC G034.43+00.24, ApJ, 630, L181

Rathborne, J. M., Jackson, J. M., \& Simon, R., 2006, Infrared Dark Clouds: Precursors to Star Clusters, ApJ, 641, 389

Rathborne, J. M., Simon, R., \& Jackson, J. M., 2007, The Detection of Protostellar Condensations in Infrared Dark Cloud Cores, ApJ, 662, 1082

Rathborne, J. M., Jackson, J. M., Zhang, Q., \& Simon, R., 2008, Submillimeter Array Observations of Infrared Dark Clouds: A Tale of Two Cores, ApJ, 689,1141

Redman, R. O., Feldman, P. A., Wyrowski, F., Côté, S., Carey, S. J., \& Egan, M. P., 2003, Interactions between a Bright Young Stellar Object and the Midcourse Space Experiment Infrared-dark Cloud G79.3+0.3: An Early Stage of Triggered Star Formation?, ApJ, 586, 1127

Shu, F. H., Adams, F. C., \& Lizano, S., 1987, Star formation in molecular clouds - Observation and theory, ARA\&A, 25, 23

Simon, R., Jackson, J. M., Rathborne, J. M., \& Chambers, E. T., 2006a, A Catalog of Midcourse Space Experiment Infrared Dark Cloud Candidates, ApJ, 639, 227

Simon, R., Rathborne, J. M., Shah, R. Y., Jackson, J. M., \& Chambers, E. T., 2006b, The Characterization and Galactic Distribution of Infrared Dark Clouds, ApJ, 653, 1325

Sridharan, T. K., Beuther, H., Schilke, P., Menten, K. M., \& Wyrowski, F., 2002, High-Mass Protostellar Candidates. I. The Sample and Initial Results, ApJ, 566, 931 
Sridharan, T. K., Beuther, H., Saito, M., Wyrowski, F., \& Schilke, P., 2005, High-Mass Starless Cores, ApJ, 634, L57

Teyssier, D., Hennebelle, P., \& Pérault, M., 2002, Radio-millimetre investigation of galactic infrared dark clouds, A\&A, 382, 624

Valdettaro, R., Palla, F., Brand, J., Cesaroni, R., Comoretto, G., di, F. S., Felli, M., Natale, E., Palagi, F., Panella, D., \& Tofani, G., 2001, Arcetri Catalog of H2O maser sources. Update. (Valdettaro+, 2001), VizieR Online Data Catalog, 336, 80845

Van der Walt, J., 2005, On the number and lifetime of 6.7-GHz methanol masers, MNRAS, 360, 153

Wang, Y., Zhang, Q., Rathborne, J. M., Jackson, J., \& Wu, Y., 2006, Water Masers Associated with Infrared Dark Cloud Cores, ApJ, 651, L125

Weintraub, D. A., 1990, A catalog of pre-main-sequence emission-line stars with IRAS source associations, ApJS, 74, 575

Wood D. O. S. \& Churchwell E., 1989a, Massive stars embedded in molecular clouds - Their population and distribution in the galaxy, ApJ, 340, 265

Wood D. O. S. \& Churchwell E., 1989b, The morphologies and physical properties of ultracompact H II regions, ApJS, 69, 831

Young, C. H., Jørgensen, J. K., Shirley, Y. L., Kauffmann, J., Huard, T., Lai, S.-P., Lee, C. W., Crapsi, A., Bourke, T. L., Dullemond, C. P., Brooke, T. Y., Porras, A., Spiesman, W., Allen, L. E., Blake, G. A., Evans, II, N. J., Harvey, P. M., Koerner, D. W., Mundy, L. G., Myers, P. C., Padgett, D. L., Sargent, A. I., Stapelfeldt, K. R., van Dishoeck, E. F., Bertoldi, F., Chapman, N., Cieza, L., DeVries, C. H., Ridge, N. A., \& Wahhaj, Z. 2004, A "Starless" Core that Isn't: Detection of a Source in the L1014 Dense Core with the Spitzer Space Telescope, ApJS, 154, 396 